\title{
Local Dispersal of Puccinia triticina and Wheat Canopy Structure
}

\author{
Lise Frezal, Corinne Robert, Marie-Odile Bancal, and Christian Lannou
}

First and fourth authors: UMR1290 BIOGER, INRA, F-78850 Thiverval Grignon, France; second and third authors: UMR EGC, INRA, F-78850 Thiverval Grignon, France. Accepted for publication 10 June 2009.

\section{ABSTRACT}

Frezal, L., Robert, C., Bancal, M.-O., and Lannou, C. 2009. Local dispersal of leaf rust and wheat canopy structure. Phytopathology 99:1216-1224.

The development of dynamic models jointly to simulate host growth and disease spread necessitates a precise description of pathogen dispersal in relation to canopy structure. In this study, we measured disease spread from a single infected leaf positioned at different heights in wheat canopies. The resulting lesion distribution was described along crop rows and over three leaf layers. The spore sources, although limited to a single leaf, nearly saturated the host surface accessible to the spores.
Most of the lesions were found within 30 to $40 \mathrm{~cm}$ of the source. The vertical position of the source influenced the lesion distribution and the steepness of the disease gradients. The leaf layer and the wheat row that contained the spore source were the most infected. Close to the source, a few heavily infected leaves produced steep disease gradients, whereas spore diffusion resulted in shallower gradients along the adjacent rows and on the other leaf layers. Depending on the precision needed, the lesion distribution can be described either at the level of leaf layers or by dispersal gradients for each row and leaf layer.

Additional keywords: wheat leaf rust.
Dispersal of foliar pathogens around a spore source has been described in many articles, sometimes for large distances (24) but most often at the spatial scale of an infected plant or group of plants $(7,9)$, or even a single leaf $(11)$. These studies give valuable insights to allow understanding of epidemic spread and to parameterize simulation models (18) but, in most cases, do not take into account the local structure of the host crop and its potential effect on disease distribution. Moreover, in order to obtain observable disease gradients, experiments are often based on strong spore sources, such as a small plot or a group of infected plants $(3,22,23)$. Because it is practically impossible to evaluate the number of lesions on all infected plants around the source, the resulting disease gradients are measured along transects and simply describe the level of disease as a function of the distance from the source. Even for studies based on point sources of spores, it is difficult to evaluate simultaneously local effects of the canopy structure and dispersal functions (2). For instance, the dispersal of Puccinia coronata uredospores from a single infected plant was precisely measured by Mundt and Leonard (17) in a field experiment but, in order to better evaluate the disease gradient, the canopy structure had to be altered. However, this was an acceptable compromise because the objective of the experiment was to parameterize a model that simulates dispersal in two dimensions on an isotropic host canopy (18).

In such cases, it may be reasonable to ignore the local structure of the host canopy, but in others not. Many crop-damage models $(4,5,21)$ use disease-severity curves specified at different canopy layers because the photosynthetic activity of the leaves depends on the radiation intercepted (10) and, thus, on their vertical position in the crop. For instance, Robert et al. (21) developed a model to simulate the effect of leaf rust (P. triticina) and Septoria tritici blotch on the development of a wheat crop. The model estimated light interception by the different canopy layers and, using the disease distribution over these layers, computed the loss

Corresponding author: C. Lannou; E-mail address: lannou@grignon.inra.fr

doi:10.1094/PHYTO-99-10-1216

(C) 2009 The American Phytopathological Society in biomass production with regard to a healthy crop. Such a model necessitates a description of the vertical distribution of the disease (over the different canopy layers) rather than horizontal gradients. Furthermore, the future development of models computing simultaneously disease dynamics and host development will require data on disease spread that match the level of detail in the host description.

Depending on the host species, the description of the crop structure may vary. The physical structure of a bean crop can be described in a hierarchical pattern (25), including leaflets, leaves, and plants, whereas this is probably irrelevant for a wheat crop. In a wheat canopy, a leaf's nearest neighbor is most probably a leaf from another plant at the same level in the canopy and not another leaf of the same plant. Moreover, a wheat plant develops a definite number of leaves before producing reproductive organs and, depending on its relative position in the canopy, each leaf contributes differently to plant growth (21). With this view, it is relevant to decompose a wheat crop into superimposed leaf layers rather than a population of separate plants. Moreover, because of cultural practices, wheat canopies are usually structured in rows.

In this article, we describe the dispersal of $P$. triticina, causal agent of wheat leaf rust, around an infected leaf. The size of the spore source was reduced to a single leaf in order to precisely identify the origin of the spores both horizontally and vertically. In addition to the distance from the source, the lesion distribution around the spore source was described according to the wheat row and the leaf layer within the canopy, in order to take into account the host structure.

\section{MATERIALS AND METHODS}

Overview. The experiment consisted of inoculating single leaves in a wheat canopy with leaf rust uredospores and, one pathogen generation later, determining the spatial distribution of the progeny lesions. The experiment was performed twice, in 2000 and 2001, at the INRA Grignon field station near Paris.

The experiment layout is described in Figure 1. Although, for practical reasons, the experiment was divided into individual 
plots, the actual experimental unit was the inoculated leaf and the square meter of surrounding wheat canopy. The individual plot size was 1.75 by $30 \mathrm{~m}$. These plots included nine wheat rows and were sown at a density of 250 seeds $/ \mathrm{m}^{2}$ with cv. Soissons. The distance between rows was $17 \mathrm{~cm}$. In a plot, we inoculated 14 leaves within the two central rows that were separated from each other by at least $2 \mathrm{~m}$. We inoculated leaves in canopy layers 1 (flag leaf), 2, or 3 (second and third leaves from the top, respectively). Each layer was inoculated in a different plot. Inoculated plots were separated by noninoculated buffer plots of the same size. A pathogen generation later, we collected all the plants within $\pm 40 \mathrm{~cm}$ from the inoculated leaf along wheat rows 0 (containing the inoculated leaf), +1 , and -1 . By definition, row -1 was downwind relative to row 0. Plants were collected in clusters every $5 \mathrm{~cm}$ along each row at $0-20 \mathrm{~cm}$ from the inoculated leaf position, and every $10 \mathrm{~cm}$ at 20 to $40 \mathrm{~cm}$ (see details below and Figure 1). All the progeny lesions present on leaves 1, 2, and 3 of these plants were counted. Because this counting operation took several hours, the collected plants were kept in plastic bags at $8^{\circ} \mathrm{C}$ to maintain leave freshness.

For each canopy layer, only a part (3 to 6) of the 14 replications was actually analyzed. Because inoculating a single leaf in a healthy crop is technically difficult and because external inoculum and local heterogeneities are likely to occur during a field experiment, we chose to inoculate as many leaves as possible (taking into account the plot dimensions) in order to obtain approximately five leaves with a large number of lesions in a locally healthy and homogeneous crop. In particular, replications for which the number of spontaneous infections was locally too high were removed (see below the details for each experiment).

Inoculations were performed by using a finger to apply a mixture of spores and talc (1:6) onto the central part of the leaves. Approximately half of the leaf surface was covered by the sporetalc mixture. A plastic bag previously sprayed with distilled water was then used to cover the leaf and attached around the stem, which was supported by a fiberglass stake. Inoculations were per-

\section{A}

\section{Position of inoculated leaves within a plot}

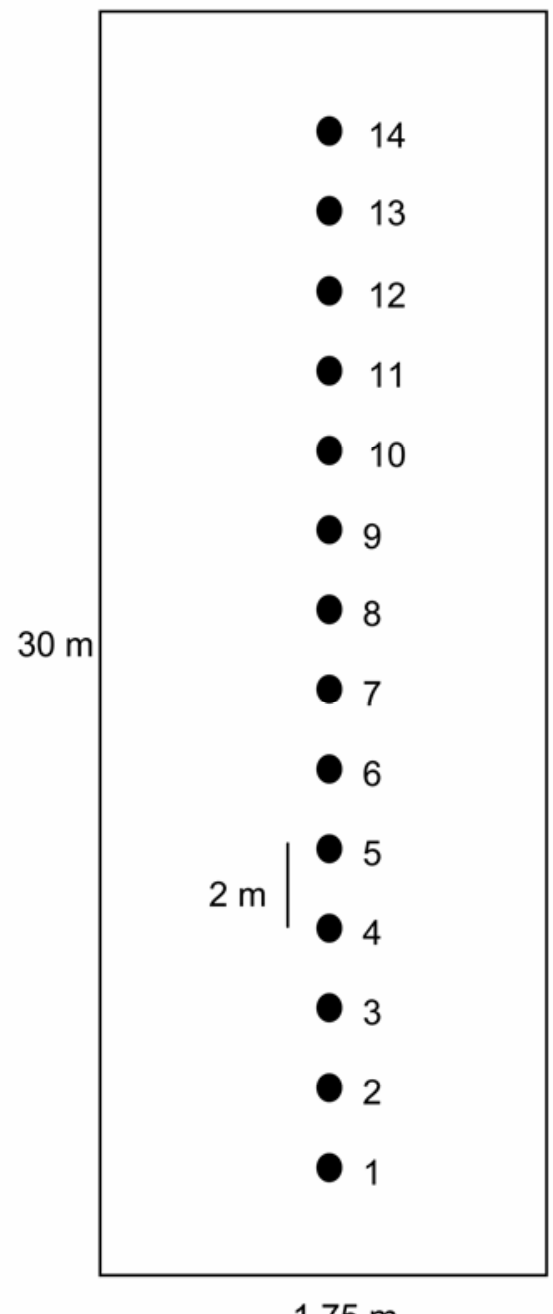

Detail of the sampling area

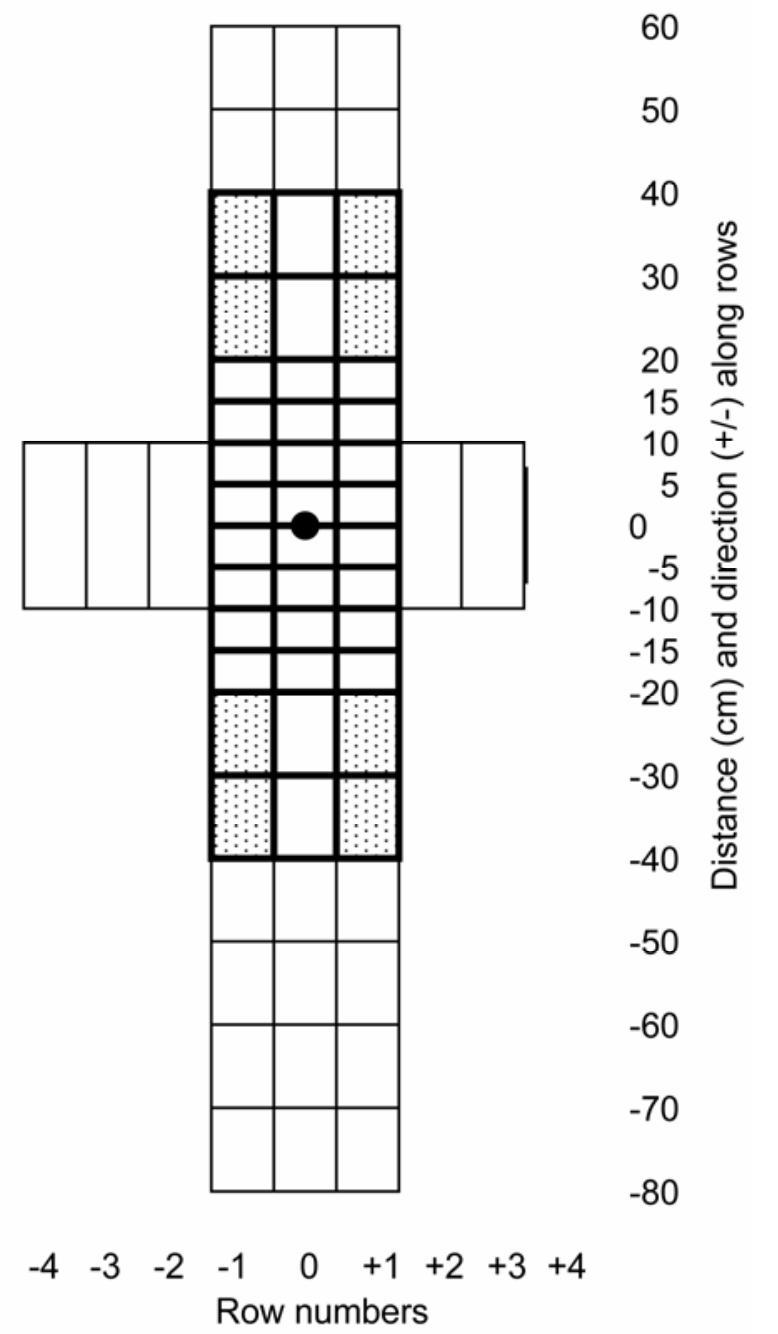

Fig. 1. Experimental design. A, Position of the inoculated leaves in the wheat plot. Each dot represents a single leaf, artificially inoculated. The plot measured 1.75 by $30 \mathrm{~m}$ and included nine wheat rows. Three different plots separated by buffer plots were used for inoculating the flag leaf and the second and third leaves from the top of the plant (leaves L1, L2, and L3, respectively). B, Sampling design for measuring the lesion distribution. The inoculated leaf is represented by a black dot. All the plants within $\pm 40 \mathrm{~cm}$ from the inoculated leaf were collected along wheat rows 0 (containing the inoculated leaf), +1 , and -1 (downwind) in 5-cm-long clusters at 0 to $20 \mathrm{~cm}$ from the inoculated leaf position, and in $10-\mathrm{cm}$ long clusters at 20 to $40 \mathrm{~cm}$. In 2000, the plants collected at distances $>20 \mathrm{~cm}$ were in row 0 only. For some replications (2001, spore source on L2), additional plants were sampled in rows 2, 3, and 4. 
formed after $1700 \mathrm{~h}$ and the bags were removed the next morning, before $1000 \mathrm{~h}$. We attempted to control, as much as possible, the external inoculum with fungicide applications (epoxiconazol, Opus; BASF). This fungicide protected the plants for an estimated time of 15 days. Spontaneous infections, considered as background noise, were regularly monitored. Fertilization and weed control were standard for the local conditions. The plots were given three nitrogen applications: 60,100 , and $80 \mathrm{~kg} / \mathrm{ha}$ at growth stages 21,30 , and 53 (26), respectively.

Hereafter, leaves 1 (flag leaf), 2, and 3 are referred to as L1, L2, and L3, respectively. The leaf rust generation resulting from artificial inoculation is referred to as g0 and the daughter lesions, resulting from the spread of asexual uredospore, as generation $\mathrm{g} 1$.

Details for each experiment. In 2000, inoculations were performed on 04 May on 14 leaves in canopy layers 1, 2, and 3. Inoculated leaves started to sporulate on 15 May and the number of lesions resulting from inoculation (g0) was counted on 19 May. The first lesions of generation $\mathrm{g} 1$ were observed on 29 May. The plants surrounding the inoculated leaves were collected on 5 June and the progeny lesions (g1) were counted in the laboratory. For distances $>20 \mathrm{~cm}$ (20 to 30 and 30 to $40 \mathrm{~cm}$ ) from the inoculated leaf position, plants were collected on row 0 only. There were three replications for L3, four replications for L2, and four replications for $\mathrm{L} 1$. To limit spontaneous infections, a fungicide treatment was applied on 22 March. Spontaneous infections were monitored on 17, 19, and 24 May: two people observed the plants surrounding the inoculated leaves ( 0 to $30 \mathrm{~cm}$ in all directions) and noted the presence or absence of leaves with $>10$ spontaneous infections. When such leaves were observed, the replication was discarded.

In 2001, inoculations were performed at different dates on the three canopy layers: on 23 April for leaf L3 (at that time, leaf L2 was almost fully expanded and leaf L1 was emerging), on 27 April for leaf L2, and on 7 May for leaf L1. Inoculated leaves started to sporulate on 09 May for L3, 11 May for L2, and 18 May for L1. The number of lesions resulting from inoculation (g0) was determined on 24 May for leaves L2 and L3 and on 31 May for L1: the inoculated leaves were collected and the number of g0 lesions was counted in the laboratory. Plants bearing daughter lesions (g1) were collected on 31 May for the spore sources on L3 (four replications), 1 June for L2 (five replications), and 11 June for L1 (five replications). All plants within $\pm 40 \mathrm{~cm}$ from the inoculated leaf position were collected on rows $-1,0$, and +1 (Fig. 1). Additional plants were collected in the plots inoculated on L2 on 3 June, at greater distances from the inoculated leaves $(>40 \mathrm{~cm}$, and up to rows \pm 4$)$. For spore sources on L1, the delay between generations g0 and g1 was longer than expected, and this was attributed to a water stress (the period was warm with a strong water deficit). Fungicide treatments were applied on 16 March on the whole experiment, and again 3 weeks before the inoculations (3 April for L2 and 13 April for L1 and the buffer plots). Spontaneous infections were monitored on 11, 15, 21, 29, and 31 May and 11 June by assessing the average number of lesions per leaf in the buffer plots. Ten groups of 10 plants, each separated by $6 \mathrm{~m}$, were observed along two buffer plots. The number of lesions was counted on each observed leaf of rank L1, L2, and L3.

Statistical analysis. The effects of host structure and inoculated leaf position in the canopy on the number of lesions per leaf were evaluated with an analysis of variance (ANOVA). The effects included in the analyses were wheat row, direction along the row, inoculated canopy layer, and target canopy layer. The distance from the inoculated leaf (spore source) along the row direction was included as a quantitative variable. Each year was analyzed separately. Lesions counts and distances were log-transformed to ensure a residual distribution compatible with the linear model. Note that the experimental design could not be fully randomized (mainly because of the timing of inoculations and fungicide treatments). In particular, the effect of the inoculated canopy level is confounded with the block effect in the ANOVA.

The disease gradients were adjusted to a power function, $y=$ $a(x+c)^{-b}$, and to an exponential function, $y=a \times e^{-b x}$, where $y$ is the number of lesions per unit area, $x$ is the distance (in meters) from the spore source position along an axis parallel to the row direction, and $c$ is a positive number that allows for prediction of a finite number of infections at the source. Parameter $b$ is a measure of the gradient steepness and parameter $a$ is proportional to the strength of the source. Here, the distance $x$ is defined within a wheat row and a gradient is described for each row. For both models, the data were log-transformed so that the parameters could be estimated from linear regression (the distance $x$ did not require transformation for the exponential function). Both functions are classically used to estimate disease gradients for plant pathogens (7). The power function was proposed by Mundt and Leonard (17) as a modification of the Gregory model (9). Although parameter $c$ had primarily no biological definition, it was found to represent approximately the radius of the spore source, and this was confirmed in other studies $(2,16)$. It has been noted that gradient estimations are sometimes better when $c$ was treated as an unknown parameter than when it was fixed as the source radius. This is expected because a function with three parameters is more flexible; however, it seems more sensible to fix parameter $c$ in order to be able to compare different estimated values of the gradient steepness $(b)$ among different epidemics or replicates $(2,16)$. For this reason, we used a fixed value $c=0.03$ in all our gradient estimations.

Estimations of disease gradients were performed with the SPLUS software (Insightful Corporation, Seattle, WA). The SAS software (SAS Institute Inc., Cary, NC), proc GLM was used for the ANOVA.

\section{RESULTS}

Lesion multiplication rate. When plotting g1 against g0 (not shown), we did not obtain a clear relationship $\left(R^{2}=0.17\right)$. Nevertheless, the slope was found to be positive $(F=4.58, P=$ $0.043)$ but small $(b=7.76)$ relative to the values of $\mathrm{g} 1$. This means that each g0 lesion on the source leaf participated to a fraction of the next generation but increasing g0 did not increase $\mathrm{g} 1$ much. The numbers of $\mathrm{g} 0$ and $\mathrm{g} 1$ lesions are given in Table 1, and the multiplication rate $\mathrm{g} 1 / \mathrm{g} 0$ is shown in Figure 2 as a function of g0. Data from leaves L3, year 2000, were not included in the analysis because of their poor quality (number of observed leaves reduced by $66 \%$ due to Septoria tritici blotch). Maximum and minimum values for the multiplication rate $\mathrm{g} 1 / \mathrm{g} 0$ were 462.1 and 10.0 , respectively. The median value was 49.2 . Given the skewed data distribution, the mean value (98.2) is poorly informative here. When considering only the values for $\mathrm{g} 0>100$, the average multiplication rate was 38.5. If the host surface available for infections was not limiting (no saturation effect), one would expect a constant multiplication rate, independent of g0. On the contrary, Figure 2 shows a clear saturation effect, with $\mathrm{g} 1 / \mathrm{g} 0$ strongly decreasing when $\mathrm{g} 0$ increases. In an attempt to better estimate the multiplication rate, a model (11) including a saturation effect was fitted to a data set restricted to year 2001 and spore sources on levels L1 and L2. The model was defined as $g_{1}=$ $\mu \times \mathrm{g}_{0}{ }^{\alpha}$, with $\mu$ representing the multiplication rate and $\alpha$ a saturation effect $(\alpha<1)$. The parameters were estimated by linear regression, after a log-transformation. The values obtained were $\mu=7056$ and $\alpha=0.09$, with an $R^{2}$ value of 0.12 . When a single value, considered as an outlier, was removed, we found $\mu=7316$ and $\alpha=0.10$, with an $R^{2}$ value of 0.32 .

Spontaneous infections. In 2001, the level of natural infections was recorded regularly in the buffer plots. On 11 May, only nine lesions were found after $1 \mathrm{~h}$ of observation. On 21 May, the 
average number of lesions per leaf was 0.08 on L1, 0.015 on L2, and 0.005 on L3. On 31 May, the level of natural infections increased to 1.03 lesion/leaf on L1 and 1.81 on L2. On L3, Septoria tritici blotch started to develop and large parts of the leaves became necrotic. On 11 June, there were, on average, 8.4 lesions per leaf on L1 and 12.8 on L2. A detailed description of spontaneous infection in the 2001 experiment is given in (11). In 2000 , the choice of the replications was based on the presence or absence of natural infections near the inoculated leaves but there was no quantification of spontaneous infections. However, because the control of spontaneous infections by fungicide was less precise and because all leaves were inoculated at the same date, the background noise was higher in 2000 relative to 2001 . This difference can be evaluated by the lesion/leaf values at 40 $\mathrm{cm}$ from the spore source (Table 2). At this distance, the number of lesions was clearly higher in 2000 than in 2001.

Lesion distribution in the canopy. The distribution of the progeny lesions on leaf layers L1, L2, and L3 and rows $-1,0$, and 1 is shown in Table 3 . In general, row 0 and the canopy layer that had been inoculated were the most infected. The main exception was for the source in layer L1 in 2000. For the spore source in L3 in the 2000 experiment, the most diseased row and layer were not those with the source leaf; however, these data were not obtained with a good precision level. Considering that the lesions directly resulted from the spore flows, at least $10 \%$ of the spores moved from leaf layer L1 to L3 or from L3 to L1. Approximately half of the spores produced on L2 remained on the same level and the rest were almost equally distributed between L1 and L3. Lesion flows between L1 and L2 are less clear because of a discrepancy between 2000 and 2001 but the sporulating leaves in L1 infected

TABLE 1. Number of parent lesions (g0), progeny lesions (g1), and multiplication rate $(\mathrm{g} 1 / \mathrm{g} 0)$

\begin{tabular}{|c|c|c|c|c|c|}
\hline $\begin{array}{l}\text { Inoculated } \\
\text { leaf }{ }^{w}\end{array}$ & Replication & Year & $\mathrm{g} 0^{\mathrm{x}}$ & $\mathrm{g} 1^{\mathrm{y}}$ & $\begin{array}{l}\text { Multiplication } \\
\text { rate }\end{array}$ \\
\hline \multirow[t]{4}{*}{ L1 } & 1 & 2000 & 203 & 16,256 & 80.1 \\
\hline & 2 & $\ldots$ & 76 & 13,047 & 171.7 \\
\hline & 3 & $\ldots$ & 260 & 9,811 & 37.7 \\
\hline & 4 & $\ldots$ & 260 & 21,711 & 83.5 \\
\hline \multirow[t]{5}{*}{ L1 } & 1 & 2001 & 668 & 13,076 & 19.6 \\
\hline & 2 & $\ldots$ & 978 & 17,946 & 18.3 \\
\hline & 3 & $\ldots$ & 75 & 12,353 & 164.7 \\
\hline & 4 & $\ldots$ & 25 & 11,553 & 462.1 \\
\hline & 5 & & 483 & 12,002 & 24.8 \\
\hline \multirow[t]{4}{*}{ L2 } & 1 & 2000 & 21 & 6,333 & 301.6 \\
\hline & 2 & $\ldots$ & 200 & 10,381 & 51.9 \\
\hline & 3 & $\ldots$ & 146 & 6,801 & 46.6 \\
\hline & 4 & $\ldots$ & 66 & 14,370 & 217.7 \\
\hline \multirow[t]{5}{*}{ L2 } & 1 & 2001 & 304 & 13,149 & 43.3 \\
\hline & 2 & $\ldots$ & 142 & 8,408 & 59.2 \\
\hline & 3 & $\ldots$ & 616 & 15,810 & 25.7 \\
\hline & 4 & $\ldots$ & 445 & 12,314 & 27.7 \\
\hline & 5 & $\ldots$ & 300 & 6,207 & 20.7 \\
\hline \multirow[t]{3}{*}{$\mathrm{L}^{\mathrm{z}}$} & 1 & 2000 & 37 & 12,610 & 340.8 \\
\hline & 2 & $\ldots$ & 96 & 6,731 & 70.1 \\
\hline & 3 & $\ldots$ & 6 & 8,010 & $1,335.0$ \\
\hline \multirow[t]{4}{*}{ L3 } & 1 & 2001 & 176 & 1,760 & 10.0 \\
\hline & 2 & $\ldots$ & 218 & 4,590 & 21.1 \\
\hline & 3 & $\ldots$ & 58 & 12,666 & 218.4 \\
\hline & 4 & $\ldots$ & 134 & 7,158 & 53.4 \\
\hline Maximum & $\ldots$ & $\ldots$ & $\ldots$ & 21,711 & 462.1 \\
\hline Mean & $\ldots$ & $\ldots$ & $\ldots$ & 11,259 & 98.2 \\
\hline Median & $\ldots$ & $\ldots$ & $\ldots$ & 12,158 & 49.2 \\
\hline Minimum & $\ldots$ & $\ldots$ & $\ldots$ & 1,760 & 10.0 \\
\hline
\end{tabular}

${ }^{\mathrm{w}}$ Leaves from three canopy layers were inoculated. L1, L2, and L3 = flag leaf and the second and third leaves from the top, respectively.

${ }^{x} \mathrm{~g} 0$ Lesions were produced by artificial inoculation.

${ }^{y} \mathrm{~g} 1$ Lesions resulted from spore dispersal from $\mathrm{g} 0$ lesions.

${ }^{\mathrm{z}}$ These values were measured with a lower precision (number of observed leaves reduced by $66 \%$ due to Septoria tritici blotch) and were not used in the statistics.
L2 at least as much as L1. It is noticeable that, in both years, the spore flows from L3 toward L2 and L1 were symmetrical with those from L1 toward L2 and L3. In 2001, a little less than half of the g1 lesions were found on the source layer, $\approx 10 \%$ on the most distant leaf layer and the remaining $(\approx 43 \%)$ on leaf layer L2. That year, row 0 was always more infected than the two adjacent rows. Row -1 tended to be more infected than row 1 and this probably resulted from prevailing winds. Data in Table 3 are average values and indicate general trends but local variability occurred at a smaller scale. For instance, when considering only distances between -10 and $+10 \mathrm{~cm}$ from the spore source and row 0 , lesions originating from a source in L1 are distributed (in percent) according to $\mathrm{L} 1=71.4, \mathrm{~L} 2=27.3$, and $\mathrm{L} 3=1.3$ instead of $\mathrm{L} 1=$ $47.9, \mathrm{~L} 2=43.7$, and L3 $=8.4$ (Table 3, 2001). These local heterogeneities are better described by the disease gradients (Fig. 3 ).

The effects of spore source position, canopy layer, row, and direction along the row on the number of lesions per leaf were evaluated with an ANOVA, with the distance from the source as a covariate. We considered the level of significance to be $\alpha=0.01$. In 2000, source position L3 was removed from the analysis because of the poor data quality. All main effects were significant $(P<0.01)$. Most of the variance was accounted for by canopy layer $(25.9 \%)$, followed by source position $(12.4 \%)$ and row

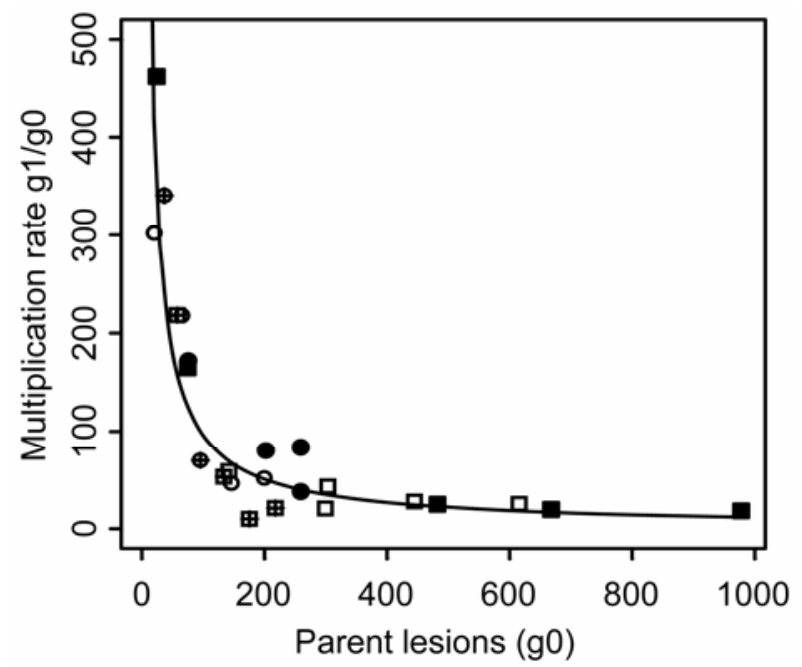

Fig. 2. Lesion multiplication rate $(\mathrm{g} 1 / \mathrm{g} 0)$ as a function of the number of parent lesions (g0) in the spore sources. L1, L2, and L3 = flag leaf and the second and third leaves from the top, respectively. Circles: 2000; squares: 2001. Solid symbols: spore source on leaf L1; empty symbols: L2; crossed: L3. A model $\log \left(\mathrm{g}_{1} / \mathrm{g}_{0}\right)=a+b \times \log \left(\mathrm{g}_{0}\right)$ was fitted to the data (continuous line, $R^{2}=0.82$ ).

TABLE 2. Number of lesions per leaf at $40 \mathrm{~cm}$ from the source and background infection $(\mathrm{BI})$

\begin{tabular}{|c|c|c|c|c|c|c|}
\hline \multicolumn{2}{|c|}{ Canopy layer ${ }^{\mathrm{X}}$} & \multirow{2}{*}{$\frac{2000^{y}}{\text { Row } 0}$} & \multicolumn{4}{|c|}{$2001^{y}$} \\
\hline Source & Target & & Row 0 & Row -1 & Row +1 & $\mathrm{BI}^{\mathrm{z}}$ \\
\hline \multirow[t]{3}{*}{ L1 } & 1 & 13.8 & 12.7 & 11.7 & 7.4 & 8.42 \\
\hline & 2 & 62.2 & 18.3 & 17.1 & 11.4 & 12.81 \\
\hline & 3 & 30.2 & 6.3 & 3.2 & 2.1 & $\ldots$ \\
\hline \multirow[t]{3}{*}{ L2 } & 1 & 16.8 & 3.1 & 2.4 & 1.4 & 1.03 \\
\hline & 2 & 24.2 & 5.8 & 3.7 & 3.2 & 1.81 \\
\hline & 3 & 6.8 & 1.7 & 1.0 & 1.1 & $\ldots$ \\
\hline \multirow[t]{3}{*}{ L3 } & 1 & 22.6 & 2.0 & 2.6 & 3.8 & 1.03 \\
\hline & 2 & 40.1 & 11.0 & 10.0 & 10.0 & 1.81 \\
\hline & 3 & 10.6 & 8.0 & 8.2 & 11.0 & $\ldots$ \\
\hline
\end{tabular}

${ }^{\mathrm{x}} \mathrm{L} 1, \mathrm{~L} 2$, and L3 = flag leaf and the second and third leaves from the top, respectively; source $=$ inoculated leaves $;$ target $=$ canopy layer on which progeny lesions were counted.

y Wheat rows: 0 contained the inoculated leaf and -1 was downwind.

${ }^{\mathrm{z}}$ Background infection measured in the buffer plots when the progeny lesions were counted. 
(12.6\%), then by direction along the rows and distance from the source $(<2 \%$ each). The number of lesions per leaf was always significantly greater on layer L2 than on L1 and L3. An interaction was detected between source position and row (Table 4) but, for both source positions, the number of lesions per leaf was not significantly different on row -1 and row 0 , and was lower on row 1 . The plants along direction -1 were more infected than along direction 1 . In 2001, all main effects were significant $(P<0.01)$ except the direction $(P=0.022)$, and there were interactions between source position and the three other factors (Table 4). Most of the variance was accounted for by canopy layer (26.8\%), followed by source position $(22.8 \%)$, distance from the source $(10.3 \%)$, and row $(5.5 \%)$. Separate analyses were performed for each source position. The interaction between source position and canopy layer was consistent with the lesion distribution described in Table 3: when the spore source was positioned on leaf L1, canopy layer L3 was significantly less infected than L1 and L2; when the spore source was on L2, the canopy layer L2 was significantly more infected than L1 and L3; when the spore source was on L3, layer L1 was less infected than L2 and L3. In all situations, row 0 was more infected than rows 1 and -1 but the differences were significant only when the spore source was on L2 (row $0 \neq$ row $-1 \neq$ row 1 ) and L3 (row $0 \neq$ row -1 ). As in 2000 , leaves along direction -1 were more infected than along direction 1 , but this difference was significant only when the spore source was on L1 and L3.

Disease gradients. The disease gradients around the spore sources are shown in Figure 3. In 2000, the disease gradients on rows 0 and -1 are comparable whereas, in 2001, the highest disease densities were restricted to row 0. In 2000, background noise resulting from the development of natural infections interfered with the experiment. The disease level at $40 \mathrm{~cm}$ from the source is an indication of the level of spontaneous infections in the canopy (Table 2). However, the gradient structure remained visible, at least for spore sources on L1 and L2. The disease distribution is much clearer in 2001 and shows steep gradients on row 0 , with the highest densities on the canopy layers that included the inoculated leaves. Exponential and power functions were fitted to the disease gradients and average values of the function parameters were calculated based on the data sets for which the $R^{2}$ values were $>0.5$ (Table 5 ). For both functions, the gradient steepness is indicated by parameter $b$. Highest values of $b$ were found for sources in L2 and row 0 . Gradients originating from L3 leaves were less pronounced. Steepness of the gradients appeared lower in 2000, probably because of the background noise.

In the 2001 experiment and for spore source in L2 only, disease was assessed on an extended number of wheat rows. The mean disease gradients perpendicularly to the rows are shown in Figure 4. It shows that only two rows away from the source $(34 \mathrm{~cm})$, the number of lesions per leaf was close to the background infection level in the plot. Fitting these data to the power function (modified Gregory model) gave average gradient values of $b=$ 1.15 on leaf layer L1 $\left(R^{2}=0.90\right), b=1.02$ on L2 $\left(R^{2}=0.87\right)$, and $b=1.28$ on L3 $\left(R^{2}=0.79\right)$. These gradients were flatter than the gradients measured along the row, with $b=1.54,1.52$, and 1.65, respectively (Table 5).

\section{DISCUSSION}

Background infection was better controlled in 2001, and the quality of the data was better that year than in 2000. Nevertheless, both years gave consistent results and showed a fast decline in the lesion density with increasing distance from the source, a strong effect of the canopy structure, and similar relative distributions of the progeny lesions on the three leaf levels of the crop.

The main difference between 2000 and 2001 in the lesion distribution was on the two wheat rows adjacent to the spore source (rows -1 and +1 ). In 2001, the row in which the spore source was positioned was always much more infected than the two others whereas, in 2000, the amount of g1 lesions was comparable in rows 0 and -1 . In 2000 and for the spore source on leaf level L2, the highest lesion density (Fig. 3A) and the steepest disease gradient (Table 5) were actually found on row -1 . Another difference between both years was that, in 2001, the g1 lesions originating from a source on L1 were evenly distributed between L1 and L2 whereas, in 2000, their number was almost twice as high on $\mathrm{L} 2$ relative to $\mathrm{L} 1$. Such differences are likely to occur between two different years because spore dispersal is strongly affected by environmental factors (9). Particularly, the strong infection of row -1 in 2000 could be attributed to the effect of dominant winds.

The level of background infection was much higher in 2000 than in 2001. This obviously impaired the quality of the 2000 data but, nevertheless, allowed measurement of disease gradients and description of the pattern of the lesion distribution in the canopy. The main effect of a high level of natural infections was probably to flatten the dispersal gradients (Fig. 3; Table 5, parameter $b$ ).

TABLE 3. Lesion distribution between canopy layers and rows

\begin{tabular}{|c|c|c|c|c|c|c|c|c|}
\hline \multirow[b]{3}{*}{ Year } & \multirow[b]{3}{*}{ Source $^{\mathrm{u}}$} & \multirow[b]{3}{*}{$n^{\mathrm{v}}$} & \multicolumn{6}{|c|}{ Progeny lesions ${ }^{\mathrm{w}}$} \\
\hline & & & \multicolumn{3}{|c|}{ Canopy layers ${ }^{\mathrm{x}}$} & \multicolumn{3}{|c|}{ Wheat rows ${ }^{\mathrm{y}}$} \\
\hline & & & L1 & $\mathrm{L} 2$ & L3 & Row -1 & Row 0 & Row 1 \\
\hline \multirow[t]{2}{*}{2000} & L1 & 4 & 27.8 & 52.6 & 19.7 & 38.8 & 43.6 & 17.6 \\
\hline & $\ldots$ & $\ldots$ & 3.4 & 6.3 & 3.3 & 6.7 & 3.7 & 6.3 \\
\hline \multirow[t]{2}{*}{2001} & L1 & 5 & 47.9 & 43.7 & 8.4 & 31.6 & 44.2 & 24.2 \\
\hline & $\ldots$ & $\ldots$ & 9.0 & 6.7 & 5.3 & 8.4 & 6.8 & 2.8 \\
\hline \multirow[t]{2}{*}{2000} & $\mathrm{~L} 2$ & 4 & 21.5 & 59.6 & 18.8 & 37.3 & 38.0 & 24.6 \\
\hline & $\ldots$ & $\ldots$ & 1.3 & 3.7 & 4.0 & 7.8 & 5.3 & 6.6 \\
\hline \multirow[t]{2}{*}{2001} & $\mathrm{~L} 2$ & 5 & 20.2 & 54.1 & 25.7 & 17.7 & 70.2 & 12.1 \\
\hline & $\ldots$ & $\ldots$ & 2.6 & 7.2 & 7.0 & 5.8 & 5.3 & 2.8 \\
\hline \multirow[t]{2}{*}{2000} & $\mathrm{~L} 3^{\mathrm{z}}$ & 3 & 23.0 & 55.5 & 21.5 & 30.7 & 22.4 & 37.0 \\
\hline & $\ldots$ & $\ldots$ & 3.1 & 2.1 & 5.1 & 9.1 & 1.8 & 7.5 \\
\hline \multirow[t]{2}{*}{2001} & L3 & 4 & 10.0 & 43.3 & 46.6 & 22.3 & 45.8 & 31.8 \\
\hline & $\ldots$ & $\ldots$ & 1.1 & 3.7 & 4.3 & 3.9 & 13.2 & 9.7 \\
\hline
\end{tabular}

"Canopy layer that was the source of spores. L1, L2, and L3 = flag leaf and the second and third leaves from the top, respectively.

v Number of replications.

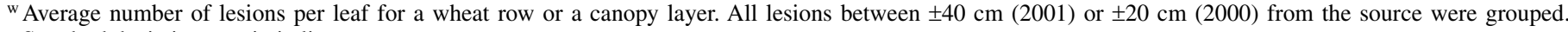

Standard deviations are in italics.

${ }^{x}$ Lesion distribution over canopy layers L1, L2, and L3.

y Lesion distribution over wheat rows; row 0 contained the inoculated leaf and row -1 was downwind.

$\mathrm{z}$ These values were measured with a lower precision (number of observed leaves reduced by $66 \%$ due to Septoria tritici blotch). 

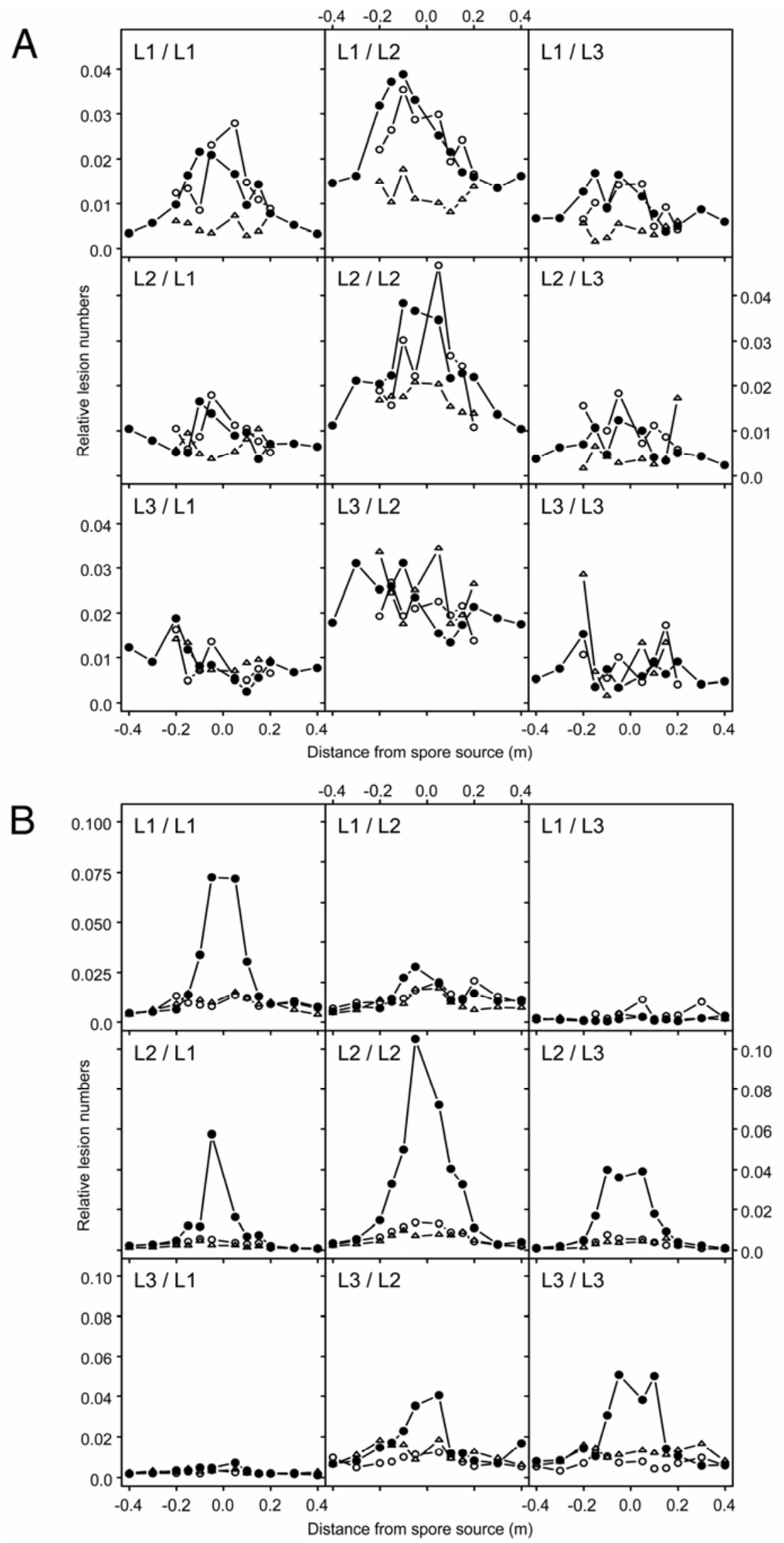

Fig. 3. Disease gradients: average number of lesions per leaf against the distance (m) from the spore source in $\mathbf{A}, 2000$ and $\mathbf{B}, 2001$. $x$-axis indicates the distance along an axis parallel to the rows, in two directions $( \pm$ ). y-axis shows relative lesion numbers: number of lesions per distance $\times$ row $\times$ canopy layer, divided by the total number of lesions produced by the source. L1, L2, and L3 = flag leaf and the second and third leaves from the top, respectively. Source and target canopy layers are indicated on the plots: L1/L2 means source layer $=\mathrm{L} 1$ and target layer $=\mathrm{L} 2$. Solid circles: row 0; empty circle: row -1 ; empty triangle: row 1 . 
Another unwanted effect that could have influenced the gradient shape is interference between the spore sources. However, considering that the spore sources were at least $2 \mathrm{~m}$ apart, and considering the gradient shape in Figure 3, it is very unlikely that

TABLE 4. Analyses of variance of the number of lesions per leaf on three leaf layers along the wheat rows and for different positions of spore sources

\begin{tabular}{lrrrc}
\hline & \multicolumn{3}{c}{ Mean } \\
Source of variation & df & square & $F$ value & $P$ value $^{\mathrm{y}}$ \\
\hline $2000^{\mathrm{z}}$ & & & & \\
Source position (S) & 1 & 53.45 & 106.93 & 0.0000 \\
Row & 2 & 25.74 & 51.48 & 0.0000 \\
Direction (dir) & 1 & 3.34 & 6.69 & 0.0099 \\
Leaf layer (L) & 2 & 62.05 & 124.13 & 0.0000 \\
Distance to source & 1 & 6.22 & 12.45 & 0.0005 \\
S $\times$ row & 2 & 3.02 & 6.05 & 0.0025 \\
Residuals & 495 & 0.50 & $\ldots$ & $\ldots$ \\
2001 & 2 & 40.35 & 58.45 & 0.0000 \\
Source position (S) & 2 & 32.43 & 46.97 & 0.0000 \\
Row & 1 & 3.61 & 5.23 & 0.0222 \\
Direction (dir) & 2 & 98.66 & 142.92 & 0.0000 \\
Leaf layer (L) & 1 & 195.25 & 282.86 & 0.0000 \\
Distance to source & 4 & 9.38 & 13.59 & 0.0000 \\
S $\times$ row & 2 & 4.68 & 6.78 & 0.0012 \\
S $\times$ dir & 4 & 76.19 & 110.38 & 0.0000 \\
S $\times$ L & 1,443 & 0.69 & 0.69 & $\ldots$ \\
Residuals &
\end{tabular}

у Only significant interactions are shown $(\alpha=0.01)$.

${ }^{\mathrm{z}}$ In 2000, source position L3 (third leaf from the top) was removed from the analysis because of the poor data quality. gradients generated by different spore sources overlap, at least at a detectable level.

Based on the counting of g0 and g1 lesions, we attempted to estimate directly the lesion multiplication rate. Lesion generation g1 was aggregated in small foci (Fig. 3), indicating that the largest part of the spores was deposited close to the source. It has been shown repeatedly that, in rust and powdery mildew epidemics, most of the spores remain within the canopy $(3,12,13)$. For instance, in an experiment using a local spore source of 10 wheat tillers infected with $P$. graminis, only $2 \%$ as many spores were trapped $10 \mathrm{~cm}$ above the canopy as were trapped $10 \mathrm{~cm}$ above the ground (23). As well, with $P$. triticina, Eversmeyer and Kramer (6) found that the spores trapped at 15 to $25 \mathrm{~cm}$ above the crop represent $<10 \%$ of the spore concentration within the crop. Therefore, we considered that the measured number of g1 lesions represented the largest part of this pathogen generation and that g1/g0 approximated the actual lesion multiplication rate in a healthy canopy.

When plotted against g0, this multiplication rate clearly showed a saturation effect for both years and the three source positions within the crop (Fig. 2). Variations in the number of g1 lesions were relatively small compared with variations in $\mathrm{g} 1 / \mathrm{g} 0$. For instance, in 2001 and canopy layer L1, the number of g1 lesions was 11,553 to 17,946 (Table 1 ), whereas g1/g0 was 18.3 to 462.1 . This could actually result from two different effects. First, it seems that, even for the smallest g0 sources (Table 1, 21 and 25 lesions), the host surface accessible to the spores was nearly saturated with progeny lesions. Increasing the number of g0

TABLE 5. disease gradients along the wheat rows and for leaf layers 1,2 , and 3

\begin{tabular}{|c|c|c|c|c|c|c|c|c|c|c|c|}
\hline \multirow[b]{2}{*}{ Year } & \multicolumn{2}{|c|}{ Canopy layers } & \multirow[b]{2}{*}{ Rows $^{\mathrm{z}}$} & \multicolumn{4}{|c|}{ Exponential model $^{\mathrm{v}}$} & \multicolumn{4}{|c|}{ Power model ${ }^{\mathrm{w}}$} \\
\hline & Source ${ }^{x}$ & Target $^{\mathrm{y}}$ & & $\log (a)$ & $b$ & $R^{2}$ & $n$ & $\log (a)$ & $b$ & $R^{2}$ & $n$ \\
\hline 2000 & L1 & L1 & -1 & -3.582 & 7.507 & 0.84 & 6 & -5.858 & 0.641 & 0.79 & 7 \\
\hline 2000 & L1 & L1 & 0 & -3.671 & 6.294 & 0.84 & 7 & -6.444 & 0.906 & 0.85 & 7 \\
\hline 2000 & L1 & L2 & 0 & -3.082 & 5.135 & 0.85 & 5 & -5.292 & 0.723 & 0.84 & 5 \\
\hline 2000 & L1 & L1 & 1 & -3.969 & 4.014 & 0.77 & 1 & -5.514 & 0.453 & 0.85 & 1 \\
\hline 2000 & L2 & L2 & -1 & -3.059 & 9.665 & 0.91 & 4 & -5.330 & 0.685 & 0.77 & 6 \\
\hline 2000 & L2 & L1 & 0 & -3.992 & 5.783 & 0.69 & 2 & -6.487 & 0.790 & 0.66 & 4 \\
\hline 2000 & L2 & L2 & 0 & -3.123 & 4.124 & 0.71 & 4 & -5.058 & 0.612 & 0.73 & 5 \\
\hline 2000 & L2 & L2 & 1 & -3.637 & 5.535 & 0.61 & 3 & -5.494 & 0.508 & 0.76 & 2 \\
\hline 2001 & L1 & L1 & -1 & -4.032 & 4.338 & 0.75 & 4 & -5.947 & 0.602 & 0.71 & 5 \\
\hline 2001 & $\mathrm{~L} 1$ & L1 & 0 & -2.869 & 7.466 & 0.78 & 8 & -6.503 & 1.176 & 0.78 & 9 \\
\hline 2001 & L1 & L1 & 1 & -3.998 & 4.319 & 0.74 & 6 & -5.932 & 0.573 & 0.64 & 8 \\
\hline 2001 & L1 & L2 & -1 & -3.704 & 4.052 & 0.67 & 5 & -5.453 & 0.516 & 0.777 & 5 \\
\hline 2001 & L1 & L2 & 0 & -3.443 & 6.463 & 0.84 & 6 & -6.099 & 0.845 & 0.82 & 7 \\
\hline 2001 & L1 & L2 & 1 & -4.062 & 4.301 & 0.86 & 6 & -5.743 & 0.525 & 0.79 & 7 \\
\hline 2001 & L2 & L1 & -1 & -4.998 & 5.815 & 0.79 & 7 & -7.619 & 0.856 & 0.81 & 8 \\
\hline 2001 & L2 & L1 & 0 & -3.585 & 10.058 & 0.79 & 9 & -8.246 & 1.541 & 0.82 & 10 \\
\hline 2001 & L2 & L1 & 1 & -5.560 & 5.563 & 0.69 & 6 & -7.922 & 0.750 & 0.73 & 8 \\
\hline 2001 & L2 & L2 & -1 & -4.102 & 5.865 & 0.84 & 9 & -6.605 & 0.787 & 0.69 & 10 \\
\hline 2001 & L2 & L2 & 0 & -2.350 & 10.360 & 0.90 & 9 & -6.999 & 1.520 & 0.81 & 10 \\
\hline 2001 & L2 & L2 & 1 & -4.546 & 4.773 & 0.75 & 10 & -6.717 & 0.685 & 0.78 & 9 \\
\hline 2001 & L2 & L3 & -1 & -5.032 & 6.747 & 0.79 & 10 & -8.015 & 0.968 & 0.77 & 10 \\
\hline 2001 & L2 & L3 & 0 & -3.262 & 10.523 & 0.81 & 10 & -8.113 & 1.650 & 0.86 & 9 \\
\hline 2001 & L2 & L3 & 1 & -5.150 & 7.447 & 0.79 & 9 & -8.302 & 1.009 & 0.77 & 9 \\
\hline 2001 & L3 & L1 & -1 & -5.051 & 6.367 & 0.86 & 3 & -8.043 & 0.935 & 0.81 & 4 \\
\hline 2001 & L3 & L1 & 0 & -4.623 & 5.517 & 0.73 & 3 & -7.437 & 0.801 & 0.67 & 3 \\
\hline 2001 & L3 & L1 & 1 & -4.685 & 6.701 & 0.80 & 1 & -7.999 & 0.774 & 0.74 & 2 \\
\hline 2001 & L3 & L2 & -1 & -4.139 & 4.378 & 0.79 & 4 & -6.152 & 0.664 & 0.79 & 4 \\
\hline 2001 & L3 & L2 & 0 & -3.198 & 6.107 & 0.82 & 5 & -5.851 & 0.804 & 0.72 & 6 \\
\hline 2001 & L3 & L2 & 1 & -3.774 & 5.954 & 0.83 & 6 & -6.208 & 0.788 & 0.90 & 6 \\
\hline 2001 & L3 & L3 & -1 & -4.783 & 3.044 & 0.71 & 2 & -6.135 & 0.512 & 0.66 & 4 \\
\hline 2001 & L3 & L3 & 0 & -3.099 & 5.645 & 0.83 & 5 & -5.978 & 0.882 & 0.74 & 7 \\
\hline 2001 & L3 & L3 & 1 & -3.878 & 5.536 & 0.78 & 5 & -5.877 & 0.631 & 0.77 & 4 \\
\hline
\end{tabular}

${ }^{\mathrm{v}}$ Equation: $y=a \times \exp (-b x)$, average values for model parameters and $R^{2}$. Only data sets $(n)$ for which $R^{2}>0.5$ were used. Note that $y$ is a relative number of lesions. For absolute values, $a$ should be multiplied by the total number of g1 lesions (11,259 on average).

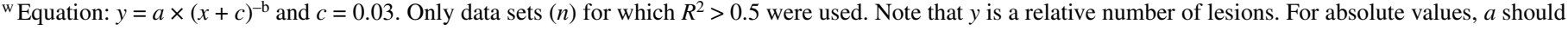
be multiplied by the total number of $\mathrm{g} 1$ lesions (11,259 on average).

${ }^{x}$ Canopy layer source of spore. L1, L2, and L3 = flag leaf and the second and third leaves from the top, respectively. In 2000 , data on L3 were of poor quality (not shown).

${ }^{y}$ Canopy layer on which progeny lesions were counted. In 2001, data for source $=$ L1 and target $=$ L3 were of poor quality (low $R^{2}$ values).

$\mathrm{z}$ Wheat row 0 contained the inoculated leaf and row -1 was downwind. 
lesions in the source had little effect on $\mathrm{g} 1$ and, thus, resulted in a lower $\mathrm{g} 1 / \mathrm{g} 0$ value. Second, it is likely that, for high lesion densities on the source leaves, the amount of spores that were produced did not increase much with the number of g0 lesions. It has been shown that leaf rust spore production is strongly density dependent (20): when lesion density is increased on a leaf, the average lesion size decreases, as does the spore production per lesion. In our experiment, both effects probably occurred: a rapid saturation of the host tissue directly accessible to the spores and a density effect on spore production by the source. Note that the accessible host surface was probably smaller than the area of green host tissue surrounding the source and might depend on the leaf geometry and relative orientation. When a model $\mathrm{g}_{1}=\mu \times \mathrm{g}_{0}{ }^{\alpha}$, was fitted to the data, we found that $\alpha$ was close to 0 , which supports the idea that source leaves with more g0 lesions did not produce many more progeny lesions than the smallest sources. Apparently, a relatively small number of lesions $(\approx 20)$ is enough to produce locally a disease focus, with a multiplication rate up to 460 lesions/lesion in a healthy crop.

It remains difficult, however, and probably impossible to estimate the actual multiplication rate of a single lesion from our data. From the 2001 data set, we found that $\mathrm{g}_{1}=7,316 \times \mathrm{g}_{0}{ }^{0.10}$ but the parameters were estimated with g0 values of 20 to 978 and with a low precision. Extrapolating to $\mathrm{g} 0=1$ does not seem reasonable here. In another article (11), we estimated autoinfection on wheat leaves infected by $P$. triticina and, using a similar model, we found that the lesion multiplication rate for autoinfection could be described with $\alpha=0.41$ and $\mu=200$ in the same 2001 experiment. Combining both models could allow estimation of the ratio between alloinfection and autoinfection, which is an important parameter for simulating epidemic development in host mixtures, but this would be valid only for leaves bearing $>20$ lesions.

The steepness of the disease gradients that were fitted to a power function were low compared with other published data. Values of parameter $b$ for gradient models based on a power function are often 1 to $4(8,9,16,17)$. In our experiment, most of the gradient values were 0.5 to 1 , except for those produced by a source on L2 and measured on row 0 , which slightly exceeded 1.5 in 2001. This is not surprising, however, because the scales of measurement are different in these studies. Such scale effects are discussed by Minogue (15), who clearly explains that comparing gradients measured over different scales of distance is hazardous. Our results could be more appropriately compared with another dispersal study based on $P$. triticina (1) in which the number of pustules was measured on trap plants at short distances from the source $(10 \mathrm{~cm}, 20 \mathrm{~cm}$, and so on). Parameter $b$ of the Gregory model was then found at 0.63 to 0.93 . There are additional reasons that make the values of $b$ we measured here difficult to compare with other published data. First, in most dispersal studies, the gradients are measured along transects and each measurement point represents the average infection level of a group of plants. For instance, Mundt and Leonard (17) measured the average numbers of progeny lesions on a group of 36 culms surrounding the source plant (in a $30-b y-30-\mathrm{cm}$ area), then on groups of 8 culms at increasing distances from the source, and finally on groups of 200 plants at greater distances. In our experiment, all the plants within $\pm 20 \mathrm{~cm}$ from the source were divided into groups of 5.7 plants, on average, for each distance value. This resulted in more detailed data at shorter distances and a greater precision close to the source (but a poor estimation of dispersal at medium distances). A second reason that can account for the shallow gradients we measured is that we decomposed the disease gradients over the different rows and leaf levels of the canopy. It seems logical to find lower values of $b$ on the rows and canopy layers that did not contain the source. Last, the lower values of $b$ found in 2000 relative to 2001 probably resulted from a flattening effect due to background infections (9).
The lesion distribution indicates that the large majority of the spores were deposited within 30 to $40 \mathrm{~cm}$ from the source. This short distance is not surprising, given the small size of the source. In published data, the distance at which g1 lesions are measured depends on the source size (7). Bainbridge and Stedman (3) detected Erysiphe graminis hordei spores at distance up to $4 \mathrm{~m}$ from a 4-m-wide infected strip of barley, and O'Hara and Brown (19) found that there was no detectable movement of $E$. graminis spores over a distance of more that $8 \mathrm{~m}$ from a barley field. In contrast, in studies based on point sources of spores, the maximal distances at which deposition is observed is of the same order of magnitude as in our experiment. MacCartney and Bainbridge (14), using E. graminis, found that spore deposit fell to $1 \%$ of its maximal value within $70 \mathrm{~cm}$ from the source. With $P$. graminis, Roelfs and Martell (23) calculated that most of the spores were deposited within $30.5 \mathrm{~cm}$ from the source.

Our experiment was not designed to compare different dispersal functions or to estimate long- or even medium-distance dispersal. It has been clearly shown that data obtained at short distances from a focus do not allow for discrimination between different dispersal functions or extrapolation of the estimated gradient to longer distances $(7,24)$.

The crop physical structure strongly influenced the local distribution of the disease. The lesion distribution among the three canopy layers clearly depended on the position of the source. In the 2001 experiment, the source layer was always the most infected, and this is consistent with results on spore fluxes within a cereal canopy (14). When the spore source was on L2, approximately half of the g1 lesions were produced on other L2 leaves and the remaining were equally distributed between L1 and L3. When the source was on L1 or L3, only $\approx 10 \%$ of the lesions were produced on the most distant canopy layer (L1 for a source on L3, and reciprocally) and the remaining were evenly distributed on the source and the nearest layers. This general pattern does not take into account local effects that were better described by the disease gradients. Most of the time, the highest values of $b$ were found on the wheat row including the spore source. The gradient

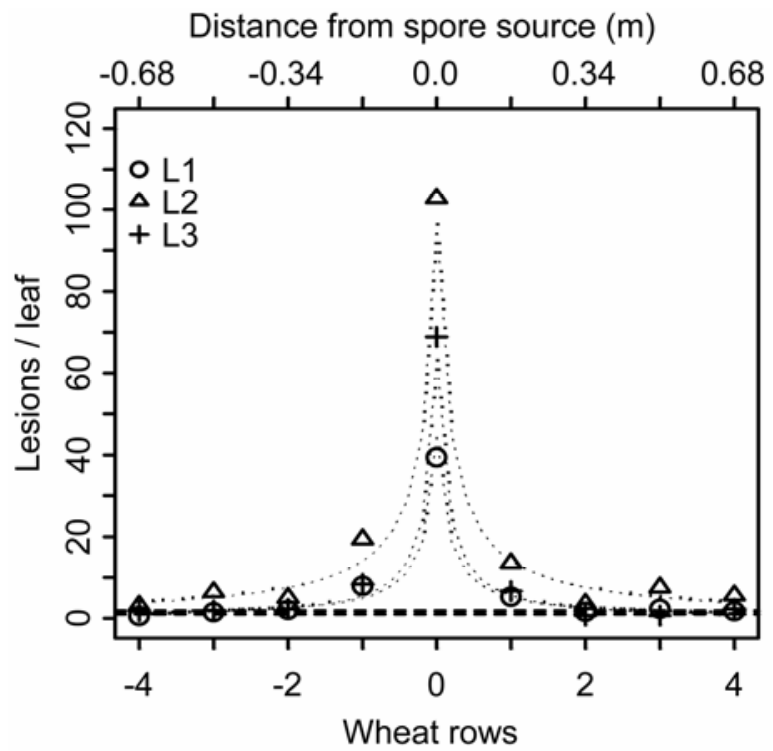

Fig. 4. Disease gradients across the wheat rows. The number of lesions per leaf is plotted against the rows and the distance $(\mathrm{m})$ to the source along an axis perpendicular to the rows. The spore source is in row $0 . \mathrm{L} 1, \mathrm{~L} 2$, and L3 = flag leaf and the second and third leaves from the top, respectively. Data are for year 2001 and spore sources on leaf L2 only. Dotted lines show adjusted gradients: $y=a(x+c)^{b}$. Horizontal dashed lines indicate the level of background infection at the time the lesions produced by sources on L1 (upper line), L2, and L3 (lower line) were counted. Circle: spore source on leaf L1; triangle: L2; cross: L3. 
steepness was clearly dependent on the vertical position of the source in the canopy. The steepest gradients along the inoculated row were for a source on $\mathrm{L} 2(\mathrm{~b} \approx 1.5)$ and the flattest were for a source on L3 $(0.8<b<0.9)$ (Table 5 , row 0$)$. When a gradient could be estimated perpendicularly to the rows (2001, L2), the value of $b(1.02<b<1.28)$ was lower than along row 0 . It seems, then, that the higher leaf density along the rows restricted the spore diffusion compared with the perpendicular direction. The disease distribution along row 0 was characterized by steep gradients and, close to the source, a few leaves heavily infected and bearing $>200$ lesions (and up to 700), whereas spore diffusion resulted in lower disease levels and shallower gradients along rows 1 and -1 .

Our main objective in this study was to describe, as precisely as possible, $P$. triticina spore dispersal in a wheat crop. We took into account the fact that wheat canopies are organized into leaf layers and plant rows. We did not consider, however, attributes of the host structure such as plant height, LAI, leaf angle, and so on that may alter the spore dispersal gradients and the spore fluxes between leaf layers.

Depending on the precision needed, the lesion distribution can be described either at the level of a leaf layer (Table 3) or by taking into consideration the dispersal gradients for each row and leaf level (Table 5; Figs. 3 and 4). Nevertheless, the clear pattern obtained with the 2001 data can be altered by environmental effects. For instance, in 2000, both rows 0 and -1 were highly infected by the g1 lesions and the lesion distribution between canopy layers L1 and L2 changed between 2000 and 2001. Depending on the strength and direction of the dominant winds during spore dispersal, deformation of disease gradients is more or less likely to occur.

\section{LITERATURE CITED}

1. Aylor, D. E. 1987. Deposition gradients of urediniospores of Puccinia recondita near a source. Phytopathology 77:1442-1448.

2. Aylor, D. E., and Ferrandino, F. J. 1990. Initial spread of bean rust close to an inoculated bean leaf. Phytopathology 80:1469-1476.

3. Bainbridge, A., and Stedman, O. J. 1979. Dispersal of Erysiphe graminis and Lycopodium clavatum spores near to the source in a barley crop. Ann. Appl. Biol. 91:187-198.

4. Bastiaans, L. 1993. Effect of leaf blast on photosynthesis of rice. 2. Canopy photosynthesis. Neth. J. Plant Pathol. 99:205-217.

5. Béasse, C., Ney, B., and Tivoli, B. 2000. A simple model of pea (Pisum sativum) growth affected by Mycosphaerella pinodes. Plant Pathol. 49:187-200.
6. Eversmeyer, M. G., and Kramer, C. L. 1980. Horizontal dispersal of urediospores of Puccinia recondita f. sp. tritici and $P$ graminis $\mathrm{f}$. sp. tritici from a source plot of wheat. Phytopathology 70:683-685.

7. Fitt, B. D. L., Gregory, P. H., Todd, A. D., Mac Cartney, H. A., and Mac Donald, O. C. 1987. Spore dispersal and plant disease gradients; A comparison between two empirical models. J. Phytopathol. 118:227-242.

8. Fried, P. M., Mackenzie, D. R., and Nelson, R. R. 1979. Dispersal gradients from a point source of Erysiphe graminis f. sp. tritici, on Chancellor winter wheat and four multilines. Phytopathol. Z. 95:140-150.

9. Gregory, P. H. 1968. Interpreting plant disease dispersal gradients. Annu. Rev. Phytopathol. 6:189-212.

10. Johnson, K. B. 1987. Defoliation and growth: a reply. Phytopathology 77:1495-1497

11. Lannou, C., Soubeyrand, S., Frezal, L., and Chadoeuf, J. 2008. Autoinfection in leaf rust dispersal. New Phytol. 177:1001-1011.

12. Last, F. T. 1955. The spore content of air within and above mildew infected cereal crops. Trans. Br. Mycol. Soc. 38:453-464.

13. Legg, B. J., and Bainbridge, A. 1978. Air movement within a crop: Spore dispersal and deposition. Pages 103-110 in: Plant Disease Epidemiology. P. Scott and A. Bainbridge, eds. Blackwell Scientific Publications, Oxford.

14. MacCartney, H. A., and Bainbridge, A. 1984. Deposition gradients near to a point source in a barley crop. Phytopathol. Z. 109:219-236.

15. Minogue, K. P. 1986. Disease gradients and the spread of disease. Pages 285-310 in: Plant Disease Epidemiology, Vol. 1. K. J. Leonard and W. E. Fry, eds. Macmillan, New York.

16. Mundt, C. C., and Leonard, K. J. 1985. A modification of Gregory's model for describing plant disease gradients. Phytopathology 75:930-935.

17. Mundt, C. C. 1989. Use of the modified Gregory model to describe primary disease gradients of wheat leaf rust produced from area sources of inoculum. Phytopathology 79:241-246.

18. Mundt, C. C., Leonard, K. J., Thal, W. M., and Fulton, J. H. 1986. Computerized simulation of crown rust epidemics in mixtures of immune and susceptible oat plants with different genotype unit areas and spatial distributions of initial diseases. Phytopathology 76:590-598.

19. O'Hara, R. B., and Brown, J. K. M. 1998. Movement of barley powdery mildew within field plots. Plant Pathol. 47:394-400.

20. Robert, C., Bancal, M.-O., and Lannou, C. 2004. Wheat leaf rust uredospore production on adult plants: Influence of leaf nitrogen content and Septoria tritici blotch. Phytopathology 94:712-721.

21. Robert, C., Bancal, M.-O., Nicolas, P., Lannou, C., and Ney, B. 2004. Analysis and modeling of effects of leaf rust and Septoria tritici blotch on wheat growth. J. Exp. Bot. 55:1079-1094.

22. Roelfs, A. P. 1972. Gradients in horizontal dispersal of cereal rust uredospores. Phytopathology 62:70-76.

23. Roelfs, A. P., and Martell, L. B. 1984. Uredospore dispersal from a point source within a wheat canopy. Phytopathology 74:1262-1267.

24. Sackett, K. E., and Mundt, C.C. 2005. Primary disease gradients of wheat stripe rust in large field plots. Phytopathology 95:983-991.

25. Willocquet, L., and Savary, S. 2004. An epidemiological simulation model with three scales of spatial hierarchy. Phytopathology 94:883-891.

26. Zadoks, J. C., Chang, T. T., and Konzak, C. F. 1974. A decimal code for the growth stages of cereals. Weed Res. 14:415-421. 